\section{THU0592 DO JUNIOR DOCTORS PRESCRIBE DISEASE MODIFYING ANTI RHEUMATIC DRUGS SAFELY?}

M.K. Nisar ${ }^{1}, \mathrm{H}$. Weller ${ }^{2} .{ }^{1}$ Rheumatology, Luton \& Dunstable University Hospital; ${ }^{2}$ Rheumatology, Luton, Luton, United Kingdom

Background: Patients with rheumatic diseases occupy over 50,000 bed days annually in the National Health Service. Adverse drug events are major causes of harm to patients in hospitals and are associated with prolonged length of stay with higher costs. National Patient Safety Agency has issued several alerts regarding DMARDs and Methotrexate overdose remains a "never event". Prescribing medications, including DMARDs for rheumatology patients, is one of the chief responsibilities of junior doctors.

Objectives: We undertook a pilot survey of junior doctors and medical students to understand their level of prescribing confidence with an aim to develop a teaching program.

Methods: Following a focus group discussion based on NPSA safety alerts and BSR DMARD monitoring guidelines, ten items were unanimously identified as core knowledge required for safe prescribing. A questionnaire was created based on these elements. Junior doctors and final year medical students were surveyed at our academic institution. Replies were compiled to ascertain their understanding of safe prescribing and troubleshooting DMARD related issues.

Results: 41 junior doctors of all grades and nine medical students contributed to the survey. Only $6 / 50(12 \%)$ felt confident in prescribing whereas $16 / 50(32 \%)$ had borderline confidence and remaining $56 \%$ felt it was beyond their expertise. Of the 41 junior doctors, 19 (46\%) had never even prescribed DMARD despite encountering such patients on the wards. Principal reasons for this included lack of confidence (40\%), paucity of knowledge (15\%) and no formal education (32\%). Questions pertaining to safe prescribing were confidently answered by only $4 / 50$ (8\%) participants.

Conclusions: To our knowledge, this is the first survey to demonstrate that there are serious shortcomings in junior doctors' understanding of safe DMARD prescribing. Inadequate training and hence poor confidence among front line medical staff remains the main cause of this issue. Despite consistent evidence suggesting that rheumatology teaching in medical schools has historically been poor and active measures taken in recent past to identify better ways to address the issues, this study highlights major knowledge gaps among everyday and future prescribers. Focused strategy and better training of junior doctors, both during and after graduation, are pivotal to providing better care for patients prescribed DMARDs during inpatient hospital stay.

Disclosure of Interest: None declared

DOI: 10.1136/annrheumdis-2017-eular.1691

\section{THU0593 QUALITATIVE ASSESSMENT OF US-GUIDED INJECTION VIDEOS PUBLISHED ON VIDEO SHARING PLATFORMS}

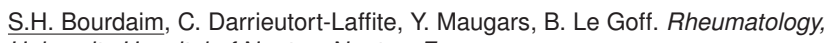
University Hospital of Nantes, Nantes, France

Background: Ultrasound (US) guided injections are becoming widespread in the treatment of rheumatic articular disorders. US allows a real time assessment of the needle progression and increase the accuracy of the injection. Video sharing platforms can be sources of information and learning material for healthcare professionals as for patients.

Objectives: We conducted this cross-sectional study to assess the quality of educational resources on US-guided articular and periarticular injections published on video sharing platforms

Methods: YouTube, Dailymotion and Vimeo were searched using predefined keywords on US-guided shoulder, elbow, wrist, hand, hip, knee, ankle, and foot injections. The videos were classified according to their source. We determined the injection site and the explanations shown for each site. We collected information on patient positioning, equipment, needle, ultrasound settings and teaching material used by the author. When demonstration was performed live in patient, the compliance with the rules of asepsis and the accuracy of the injection were evaluated. Overall, videos were evaluated for quality on a 5-point ordinal global quality scale (GQS) (from $1=$ poor quality to $5=$ excellent quality). Results are given as median (min-max).

Results: We found 69979 results with the keywords. We screened 2802 videos by titles and included 153 videos (10.05 hours). Most of videos were published on Youtube $(92.2 \%)$ and $82.4 \%$ included oral explanation. $53.6 \%$ videos were published by medical advertisement or profit companies and only $9.2 \%$ videos by university, professional organization or physician group. Among the $41.2 \%$ videos showing live demonstration of injection on the patient only $25.4 \%$ followed the strict rules of asepsis. When the videos included US cineloops of injection, $10.4 \%$ of them were outside the target. Very few videos gave details about information on the pathophysiology of the disease $(6.5 \%)$, the risk $(0.7 \%)$, the benefice $(11.2 \%)$ or the products used for the injection (58.2\%). Overall, $3.3 \%$ of the videos were classified as "Excellent quality" on the GQS, 24.2\% as "Good quality", 23.5\% as "Moderate quality", 34.6\% as "Generally poor quality" and 14,4\% "Poor quality". We compared the characteristics of the good quality videos (GQS score $\geq 4$ ) versus the one rated $\leq 3$. Better quality videos were longer $(1.3 \mathrm{~min}((0.07-1.05)$ versus $3.62(0.32-40.43) \mathrm{min}$ ), had oral explanation (95\% versus $77 \% ; p=0.008$ ). They more frequently showed the clinical and US site of injection and accurately reached their target $(90 \%$ versus $68 \%)$. They were significantly more viewed (m2719 (13-80195) versus 856 (11-60174); $\mathrm{p}=0.026)$ and more liked $(1(0-80)$ versus $7(0-58)(p=0.002))$. However, some poor quality videos had more than 60000 views. Quality of the video created by medical advertisement or profit companies were not different from those coming from university but significantly better than those from individual physician or with unknown origin $(p=0.011)$.

Conclusions: Our study reveals a generally a low quality of US guided learning videos available on the most popular video sharing platforms. We observed a lack of information on the treatment, its risks and benefits. Strict aseptic techniques are rarely followed and the injection can be outside the target. Finally, we identified characteristics associated with the quality of the video that can be used to improve their educational impact in the future.

Disclosure of Interest: None declared

DOI: 10.1136/annrheumdis-2017-eular.6396

\section{THU0594 DEVELOPING THE KOREAN LEEDS SATISFACTION QUESTIONNAIRE (KOREAN-LSQ) IN RHEUMATOID ARTHRITIS: A CROSS-CULTURAL VALIDATION USING RASCH ANALYSIS}

S.-K. Cho ${ }^{1}$, D. Kim ${ }^{1}$, S.J. Cha ${ }^{1}$, D. Yoo ${ }^{1}$, S.-H. Kim ${ }^{2}$, J. Hill ${ }^{3}$, M. Ndosi ${ }^{4}$, Y.-K. Sung ${ }^{1}$. ${ }^{1}$ Department of Rheumatology, Hanyang University Hospital for Rheumatic Diseases, Seoul; ${ }^{2}$ Department of Measurement and Evaluation of Physical Education, Chungbuk National University, Cheongju, Korea, Republic Of: ${ }^{3}$ Harrogate, Harrogate; ${ }^{4}$ Centre for Health and Clinical Research, University of the West of England, Bristol, United Kingdom

Background: The Leeds Satisfaction Questionnaire (LSQ $)^{1}$ is a patient-completed questionnaire designed to measure satisfaction among patients attending a rheumatology outpatient clinic. It was originally developed in the UK and it comprises 45 items grouped into 6 subscales: general satisfaction, information, empathy, technical competence, attitude, access and continuity.

Objectives: To undertake cross-cultural adaptation and validation of the LSQ in RA for use in Korea.

Methods: The adaptation of the LSQ from English into Korean was established according to guideline of cross-cultural adaptation of self-report measures. ${ }^{2}$ Patients with RA were then recruited from an outpatient clinic of a university hospital in South Korea and Cross-cultural validation of the Korean-LSQ was carried out by Rasch analysis using the WINSTEPS program. Model data fit was determined by Infit and Outfit statistics $(\geq 0.50$ and $\leq 1.50)$. If the values of the Infit and Outfit are 1.00, the observed score perfectly fit the expected model. The unidimensionality of the scale was determined by separation index $(\mathrm{SI} \geq 2.00)$ and reliability $(\mathrm{RI} \geq 0.80)$.

Results: An adequate conceptual equivalence was achieved following the adaptation process. The dataset for validation comprised 125 patients, 103 $(82.4 \%)$ of whom were female, mean (SD) age $=47.2$ (12.5) and disease duration $=52.8$ (69.4) months. Forty-item of the 45 items had acceptable fit statistics (individual item data not shown). The individual items overall had good separation index and reliability $(\mathrm{SI}=5.98$ and $\mathrm{RI}=0.97$ ). Analysis of the 6 subscales of the Korean-LSQ resulted in good fit to the Rasch model (Table 1), high internal consistency and unidimensionality (Person $\mathrm{SI}=2.31$ and $\mathrm{RI}=0.84$; item $\mathrm{SI}=5.44$ and $\mathrm{RI}=0.97$ ).

Table 1. Fit statistics for testlets

\begin{tabular}{lcccccc}
\hline LSQ subscales (testlets) & \multicolumn{2}{c}{ Infit } & & \multicolumn{2}{c}{ Outfit } & Point-biserical correlation \\
\cline { 2 - 3 } & MNSQ & ZSTD & & MNSQ & ZSTD & PTMEA CORR. \\
\hline LSQ_general & 1.12 & 0.90 & & 1.12 & 0.70 & 0.80 \\
LSQ_information & 1.05 & 0.40 & & 1.05 & 0.30 & 0.64 \\
LSQ_empathy & 0.64 & -3.30 & & 0.59 & -2.80 & 0.79 \\
LSQ_technical competence & 1.06 & 0.40 & & 1.05 & 0.30 & 0.77 \\
LSQ_attitude & 1.06 & 0.50 & & 0.91 & -0.40 & 0.74 \\
LSQ_access\&continuity & 1.05 & 0.50 & & 1.08 & 0.60 & 0.76 \\
\hline
\end{tabular}

$\mathrm{MNSQ}=$ mean-square; For model fit: MNSQ lie within $\geq 0.50$ and $\leq 1.50)$.

Conclusions: Fit to the Rasch model confirmed that the construct validity, reliability, and unidimensionality of the Korean-LSQ were preserved after the adaptation process. The Korean LSQ is a valid and reliable tool for measuring satisfaction with care among patients with RA in South Korea.

References:

[1] Hill J, et al. Survey of satisfaction with care in a rheumatology outpatient clinic. Ann Rheum Dis. 1992; 51(2): 195-7.

[2] Beaton DE, et al. Guidelines for the process of cross-cultural adaptation of self-report measures. Spine. 2000; 25(24): 3186-91.

Acknowledgements: This research was supported by a grant from the Korea Health Technology R\&D Project through the Korea Health Industry Development Institute (KHIDI), funded by the Ministry of Health \& Welfare, Republic of Korea (grant number: HI16C0061).

Disclosure of Interest: None declared

DOI: 10.1136/annrheumdis-2017-eular.4799 